\title{
Detection of Pre-Impact Falls from Heights Using an Inertial Measurement Unit Sensor
}

\author{
Youngho Kim *, ${ }^{\star}$, Haneul Jung ${ }^{+}(\mathbb{D}$, Bummo Koo, Jongman Kim, Taehee Kim and Yejin Nam \\ Department of Biomedical Engineering, Yonsei University, Wonju 26493, Korea; \\ hanul1219@ybrl.yonsei.ac.kr (H.J.); bmk726@ybrl.yonsei.ac.kr (B.K.); jmkim0127@ybrl.yonsei.ac.kr (J.K.); \\ kth940923@ybrl.yonsei.ac.kr (T.K.); namyj1007@ybrl.yonsei.ac.kr (Y.N.) \\ * Correspondence: younghokim@yonsei.ac.kr; Tel.: +82-33-760-2859 \\ + These authors contributed equally to this work.
}

Received: 7 August 2020; Accepted: 16 September 2020; Published: 20 September 2020

\begin{abstract}
Many safety accidents can occur in industrial sites. Among them, falls from heights (FFHs) are the most frequent accidents and have the highest fatality rate. Therefore, some existing studies have developed personal wearable airbags to mitigate the damage caused by FFHs. To utilize these airbags effectively, it is essential to detect FFHs before collision with the floor. In this study, an inertial measurement unit (IMU) sensor attached to the seventh thoracic vertebrae (T7) was used to develop an FFH detection algorithm. The vertical angle and vertical velocity were calculated using the inertial data obtained from the IMU sensor. Forty young and healthy males were recruited to perform non-FFH and FFH motions. In addition, experiments using a human mannequin and dynamics simulations were performed to obtain FFH data at heights above $2 \mathrm{~m}$. The developed algorithm achieved $100 \%$ FFH detection accuracy and provided sufficient lead time such that the airbags could be inflated completely before collision with the floor.
\end{abstract}

Keywords: falls from heights; wearable airbag; complementary filter; inertial measurement unit; detection algorithm

\section{Introduction}

Industrial and construction sites have hazardous working environments where many labor-intensive activities are performed, and several accidents can occur. Accidents in industrial sites include falls, occupational accidents, falls from heights (FFHs), fractures, and crush injury. Among these, falls and FFHs occur most frequently. According to the Korea Industrial Accident Analysis Data for 2018 [1], falls and FFHs account for $18.65 \%$ and $15.21 \%$ of the safety accidents that occur in industrial sites, respectively. In particular, the number of fatalities from FFHs was 376, accounting for one-third of the total fatalities in industrial sites. In 2014, Christina et al. [2] reported that FFHs constitute the second most common cause of work-related injuries and fatalities, resulting in 300,000 injuries and 818 traumatic deaths in the US. According to data from the Bureau of Labor Statistics [3], the number of fatal injuries due to FFHs increased by 26\% from 2011 to 2016 . The Health and Safety Executive Annual Statistics [4] reported that 40 of the 147 industrial site deaths in Great Britain in 2018/2019 were due to FFHs, accounting for the highest rate of deaths in industrial sites. The International Labour Organization [5] also mentioned that the fatality rate observed for FFHs is the highest among industrial accidents, and this issue should be handled carefully.

Thus, to prevent safety accidents such as FFHs in industrial sites, various training programs have been implemented to provide safety training to workers and site managers. Kaskutas et al. [6] conducted $8 \mathrm{~h}$ safety training sessions that included safety communication and fall protection for foremen and apprentices and evaluated the effectiveness of the training sessions through worksite 
observational audits and surveys. Consequently, the number of unsafe motions was reduced, and the number of times FFH prevention regulations were followed increased. Marin et al. [7] implemented a $5 \mathrm{~h}$ safety training program that included FFH prevention, silica exposure, leadership, communication, and safety planning for 118 workers. Six months later, the program was evaluated by Leaders in Safe Construction through surveys. The survey results showed that $58 \%$ of educated people were able to communicate effectively, $52 \%$ were more responsible for maintaining safety, and $62 \%$ said the program was effective in preventing safety accidents. However, despite efforts such as conducting safety training programs, the number of FFHs has still increased $[3,8,9]$.

The recommended physical measures to prevent FFHs include installing guardrails, covers, and safety nets and wearing personal fall arrest systems (PFASs) [10]. Chi et al. [11] analyzed various FFHs according to gender, age, working periods, and company sizes and reported that guardrails and safety nets can help prevent FFHs and minimize the damage caused by FFHs. Bobick et al. [12] evaluated the safety of two commercially available guardrails based on the criteria provided by the Occupational Safety and Health Administration and determined that the guardrails meet the safety requirements for FFH prevention. However, guardrails, covers, and safety nets are mainly installed on the outskirts of the workplace; consequently, these measures cannot prevent FFHs caused by roof or scaffold collapses within the workplace. PFASs can be used anywhere in the workplace by hanging their hooks; however, suspension trauma [13] can occur as a result of the hanging rope, and PFASs cannot prevent FFHs caused by the collapse of the workplace itself. In addition, PFASs can interrupt the movements of workers, and they do not function effectively at heights below $15 \mathrm{ft}$ [14].

To address the limitations of existing prevention methods, some studies have proposed the use of personal wearable airbags to mitigate the impact caused by FFHs on the human body [15-18]. The airbags were usually worn as clothes but were inflated to protect the human body like car airbag when dangerous situations were detected. These wearable airbags offer superior impact attenuation effects compared with commercially available protective pads [19]. Detecting FFHs before collision with the floor is essential for inflating these airbags to utilize them effectively. FFHs can be detected by either using visual devices, such as cameras, or using wearable sensors. Han et al. [20] used a Microsoft Kinect sensor and an iPi Soft Motion Capture solution and captured $90.91 \%$ of the unsafe movements performed when climbing a ladder. Shrestha et al. [21] obtained video data using high-resolution closed-circuit television (CCTV) cameras installed in the workplace. They tracked the locations of workers and determined whether the workers wore a hard hat. The system was designed to send a warning alarm when it was determined that a worker was not wearing a hard hat. Fang et al. [22] attempted to collect image data from the workplace using the mask-region-based convolution neural network and identified whether the workers moved on structural supports. The system obtained a $90 \%$ precision rate and a $75 \%$ recall rate. However, systems that use visual devices are not effective because workers might be obscured by various structures in the workplace. In addition, existing FFH detection systems that use visual devices are difficult to be applied in wearable airbags because these systems detect dangerous situations with a high probability of FFHs occurring, instead of detecting the actual FFHs.

Only a few studies have been reported on FFH detection with wearable sensors. Dogan et al. [23] performed free-fall experiments of an accelerometer. They classified FFH using root mean square (RMS) values of acceleration and calculated the fall height with an error of $10.8 \%$ using falling time. Yang et al. [24] attached an inertial measurement unit (IMU) to the waist of two participants; classified FFH and non-FFH motions such as walking, squatting, standing, losing balance, and near-fall activities on a steel frame using a support vector machine (SVM); and reported an FFH detection accuracy of $93.8 \%$. Dzeng et al. [25] used RMS values of acceleration from the smartphone to detect FFH. Four experimental participants performed some motions during tiling jobs, such as squatting, standing, and falling. They obtained $100 \%$ sensitivity and $96.1 \%$ specificity for FFH detection. However, the number of participants and experimental motions was not sufficient to objectively evaluate FFH 
detection systems in previous studies using wearable sensors. Moreover, the systems used the data at collision period and thus could not be for pre-impact FFH detection.

Many studies have been conducted for the detection of pre-impact falls of the elderly. In addition, there are many public datasets available online for fall detection systems (UMAFall [26], SisFall [27], and UP-Fall [28]). Pre-impact fall detection systems have been developed based on machine learning (ML) and threshold-based approaches. Tong et al. [29] presented a hidden Markov model (HMM)-based approach to detect and predict fall events by using the upper trunk's triaxial accelerations obtained from eight healthy young student volunteers. They acquired a lead time of $200-400 \mathrm{~ms}$ and $100 \%$ accuracy. Zhen et al. [30] calculated the RMS of acceleration and angle using inertial data from the waist and developed a pre-impact fall detection algorithm using an SVM. The algorithm obtained $99 \%$ sensitivity, 96.5 specificity, and an average lead time of $268 \mathrm{~ms}$. In general, ML-based fall detection algorithms showed good performance, but it took considerable time to extract features and to train classifiers. Therefore, it is difficult to use an ML-based pre-impact fall detection algorithm in real time.

On the other hand, for the threshold-based algorithms, Wu et al. [31] calculated the vertical velocity using inertial data obtained from the front waist and developed a pre-impact fall detection algorithm. Their algorithm achieved 100\% sensitivity, three false alarms (the number of non-falls detected as falls), and a lead time of over $70 \mathrm{~ms}$. Nyan et al. [32] also developed a threshold-based pre-impact fall detection algorithm using angles calculated from the torso and thigh (with two IMUs), as well as the correlation coefficients of the torso and thigh angles. The algorithm achieved $100 \%$ specificity, $95.2 \%$ specificity, and a lead time of $700 \mathrm{~ms}$. Sabatini et al. [33] estimated the vertical velocity and height change using an IMU with a barometric altimeter to detect fall and achieved $80 \%$ sensitivity, $100 \%$ specificity, and an average lead time of $157 \mathrm{~ms}$. Jung et al. [34] performed experiments on 30 healthy young males and obtained inertial data from the back waist. A fall detection algorithm was developed using acceleration RMS, angular velocity RMS, and vertical angle (VA). They achieved $98.33 \%$ accuracy and a lead time of $280.25 \pm 10.29 \mathrm{~ms}$ with their own data and $92.4 \%$ accuracy with SisFall public datasets.

Public datasets for the detection of fall of the elderly exist, and some previous studies detected pre-impact falls of the elderly with good performance. However, it is necessary to develop an algorithm for the detection of FFHs in worksites, which are different from falls of the elderly. FFH in worksites occurring from great heights is more fatal than fall of the elderly. In industrial sites, harsh motions such as shoveling and pickaxing and jump motion from relatively low heights are performed. Therefore, algorithms for the detection of fall of the elderly would not work in the detection of FFH in worksites.

In this study, a pre-impact FFH detection algorithm that can be applied in wearable airbags was developed to overcome the limitations of existing systems. Forty healthy young males participated in two types of experiments in which they performed various non-FFH motions and one FFH motion. In addition, in order to obtain inertial data on FFHs from great heights that cannot be used on humans owing to safety concerns, we conducted experiments using a human mannequin and dynamics simulations. The vertical angle (VA) and vertical velocity (VV) were calculated using the inertial data from the experiments and simulations, and were used as parameters in the FFH detection algorithm. The VA was calculated using a complementary filter with a proportional integral (PI) controller. The VV was calculated by integrating the vertical acceleration obtained using the Euler angle transformation of the three-axis acceleration data.

\section{Materials and Methods}

\subsection{Experimental Participants}

Two types of experiments involving humans were conducted to obtain inertial data. Forty healthy young male students from Yonsei University participated in the experiments. Among the 40 participants, 20 (age: $24.8 \pm 1.4$ years; height: $174.1 \pm 4.9 \mathrm{~cm}$; weight: $73.7 \pm 10.1 \mathrm{~kg}$ ) participated in Experiment 1, and 20 (age: $25.8 \pm 2.0$ years; height: $173.5 \pm 6.1 \mathrm{~cm}$; weight: $76.6 \pm 13.0 \mathrm{~kg}$ ) 
in Experiment 2. The Yonsei University Research Ethics Committee (1041849-201911-BM-166-01, 1041849-202004-BM-042-02) approved the experiments, and they were conducted after obtaining written consent from all the participants.

\subsection{Equipment}

An MPU-9250 [35] (InvenSense, San Jose, CA, USA) sensor capable of measuring three-axis acceleration $( \pm 16 \mathrm{~g})$, angular velocity $\left( \pm 2000^{\circ} / \mathrm{s}\right)$, and magnetism $( \pm 4800 \mu \mathrm{T})$ was used as the IMU sensor. An nRF52832 chip (Nordic Semiconductor, Trondheim, Norway) was used to receive the inertial data from MPU-9250 through I2C communication and transmit the same to a PC via radio frequency (2.4 GHz) communication. LabVIEW (National Instruments, Austin, TX, USA) was used to save the data on a PC as a .csv file. A webcam (SPC-A1200MB, Samsung, Seoul, Korea) and a 3D motion capture system (Vicon Motion Systems Ltd., Oxford, UK), which were synchronized with the IMU sensor sampled at $100 \mathrm{~Hz}$, were used to record the video and marker data.

\subsection{Experimental Procedures}

The IMU sensor was positioned on the seventh thoracic vertebrae (T7) (Figure 1). The axes of the IMU were set such that the anteroposterior was along the x-axis, mediolateral along the y-axis, and superoinferior along the z-axis.

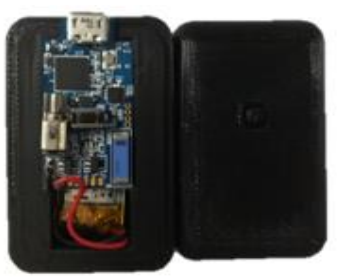

(a)

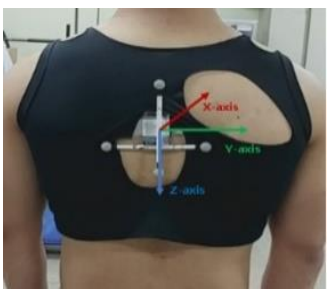

(b)

Figure 1. (a) IMU and (b) sensor position.

In Experiment 1, the participants performed nine types of non-FFH motions and one FFH motion, three times each (Table 1). In Experiment 2, the participants performed six types of non-FFH motions, in addition to the motions performed in Experiment 1 (Table 2). Figure 2 shows some of the motions performed in the experiments.

Table 1. List of motions in Experiment 1.

\begin{tabular}{cll}
\hline & \multicolumn{2}{c}{ Motions } \\
\hline & Siting quickly and getting up & Lifting (front) \\
& Siting on the floor and getting up & Lifting (back) \\
Non-FFH motions & Climbing up and down stairs & Lifting (side) \\
& Climbing up and down a ladder & $0.7 \mathrm{~m}$ jump \\
& Working with a pickaxe & \\
\hline FFH motion & $0.7 \mathrm{~m}$ forward FFH & \\
\hline
\end{tabular}

Table 2. List of additional motions in Experiment 2.

\begin{tabular}{ll}
\hline \multicolumn{1}{c}{ Motions } \\
\hline & Walking on a beam \\
& Walking on a beam with luggage in one hand \\
Shoveling & Stretching \\
& Climbing up and down a scaffold \\
& Moving up and down in an elevator \\
\hline
\end{tabular}




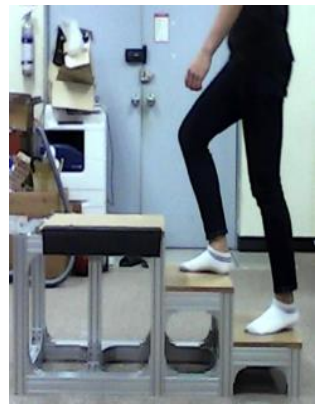

(a)

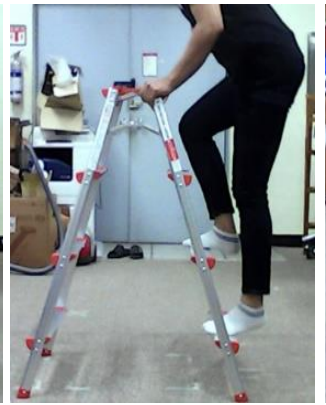

(b)

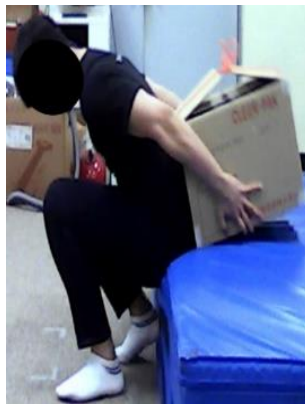

(c)

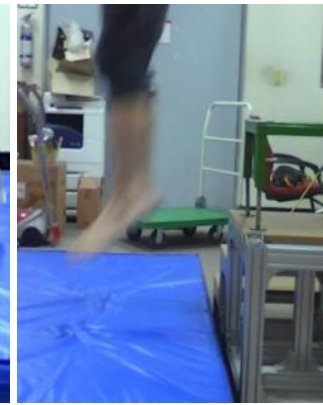

(d)

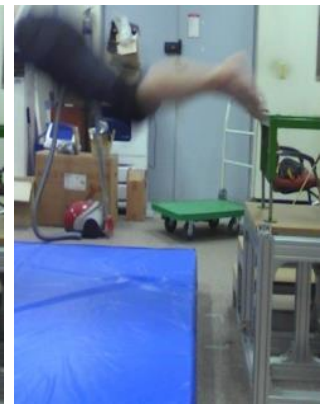

(e)

Figure 2. Examples of experimental motions: (a) climbing up and down stairs, (b) climbing up and down a ladder, (c) lifting (back), (d) $0.7 \mathrm{~m}$ jump, and (e) $0.7 \mathrm{~m}$ forward FFH.

Experiments 1 and 2 were performed to obtain data to develop an FFH detection algorithm and evaluate the algorithm, respectively. We divided the experiments into two in order to evaluate the developed algorithm with the new data that were not used in the algorithm development process and with the new motions' data that were not included in Experiment 1.

\subsection{Mannequin Experiments}

In experiments involving humans, it was not possible to perform FFH motions at great heights for safety reasons. Thus, to obtain inertial data on FFHs from great heights, we used a human mannequin (Madamade, Gyeonggi-do, Korea) (height: $180 \mathrm{~cm}$; weight: $10 \mathrm{~kg}$ ) made of soft and durable knit fabric to withstand the impact. Motions involving $0.7 \mathrm{~m}$ jump and $0.7 \mathrm{~m}$ forward FFH were performed to compare the data obtained using the mannequin with those obtained from humans. Since the mannequin cannot jump, the $0.7 \mathrm{~m}$ jump was replaced by a vertical free-fall motion at a height of $0.7 \mathrm{~m}$. Then, vertical and forward FFH motions were performed at 2, 2.5, and $3 \mathrm{~m}$. All the motions were performed five times. The IMU sensor was positioned as in the experiments involving humans. Figure 3 shows the mannequin used in the experiments, the position of the sensor, and examples of the mannequin experiments.

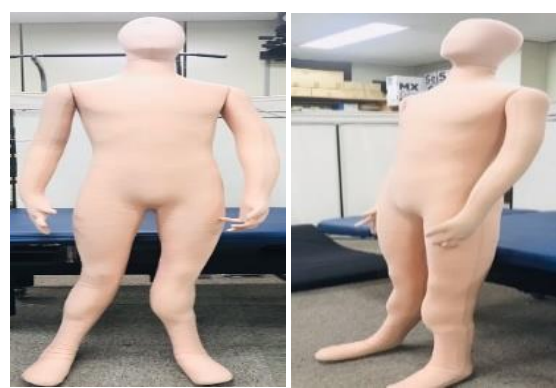

(a)

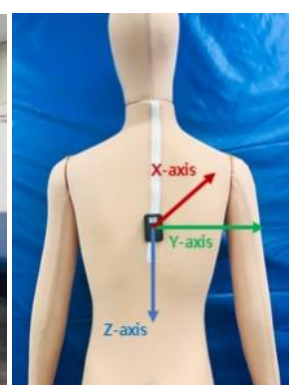

(b)

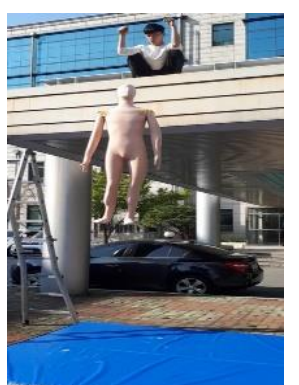

(c)

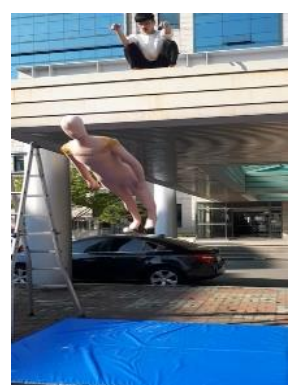

(d)

Figure 3. (a) Mannequin used in the experiments, (b) sensor position, (c) vertical FFH, and (d) forward FFH.

\subsection{Dynamics Simulations}

FFH motions were performed at great heights in the mannequin experiments; however, it was difficult to perform the FFH motions observed in actual industrial sites due to limited space and lack of equipment. Therefore, dynamics simulations were performed to obtain data on the actual FFH motions in industrial sites. Pam-Crash (ESI Group, Paris, France) and a human articulated rigid body (HARB) model (height: $170 \mathrm{~cm}$; weight: $80 \mathrm{~kg}$ ) were used. Motions involving $0.7 \mathrm{~m}$ jump and $0.7 \mathrm{~m}$ forward FFH were performed to compare the data obtained from dynamics simulations with those obtained from experiments involving humans. Since the HARB model could not jump, the $0.7 \mathrm{~m}$ jump was replaced by a vertical free-fall motion at a height of $0.7 \mathrm{~m}$. 
Zlatar et al. [36] analyzed 114 types of FFHs according to height, situation, and degree of injury. They reported that the most frequent FFHs occurred at heights between 3 and $6 \mathrm{~m}$, and many FFHs occurred when working on scaffolds, roofs, and ladders. Dynamics simulations were performed based on these findings to obtain data on FFH motions when working on scaffolds, roofs, and ladders at heights of $3,4.5$, and $6 \mathrm{~m}$. The inertial data were obtained at the same positions as those in the experiments involving humans. Figure 4 shows the motions in the dynamics simulations.

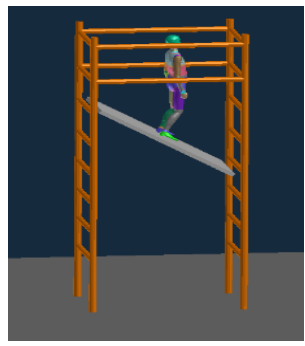

(a)

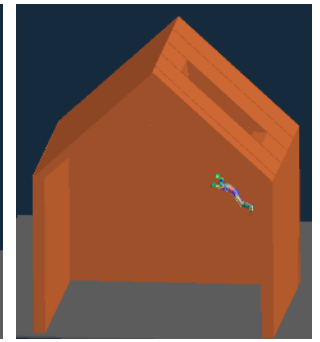

(b)

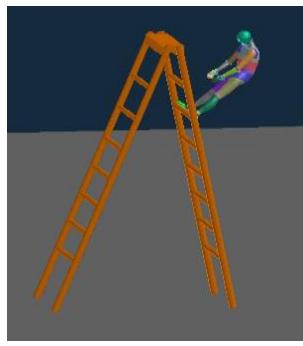

(c)

Figure 4. FFH motions in the dynamics simulations: (a) scaffold FFHs, (b) roof FFHs, and (c) ladder FFHs.

\subsection{Data Analysis}

MATLAB R2019a (MathWorks Inc., Natick, MA, USA) was used to analyze the experimental data and to develop and evaluate the FFH detection algorithm. A $5 \mathrm{~Hz}$ low-pass filter was used to filter the inertial data obtained from the IMU sensor to remove high-frequency noise.

The VA was calculated using a complementary filter with a PI controller [34] to minimize integration errors and the effect of external impacts. Figure 5 shows the flowchart of a complementary filter with the PI controller. The difference between the VA values calculated using the complementary filter with the PI controller and those obtained from the 3D motion capture system was $5.2 \pm 3.2^{\circ}$.

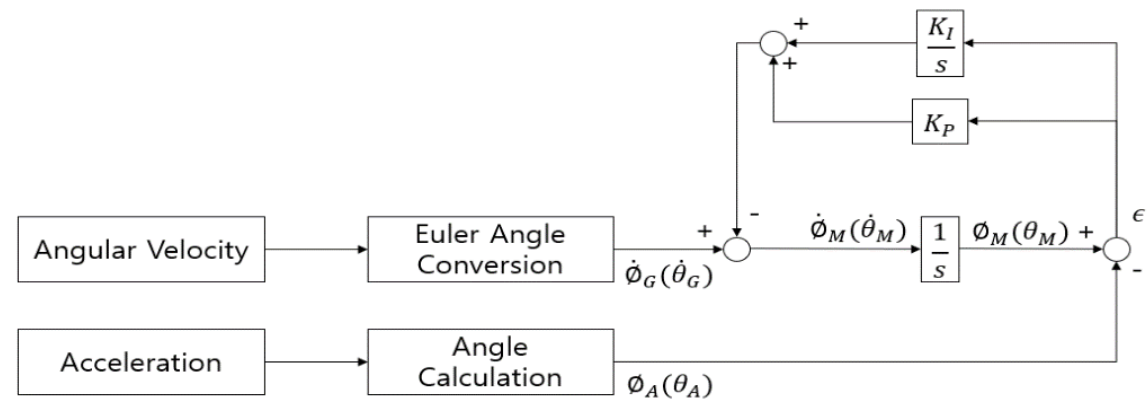

Figure 5. Vertical angle (VA) calculation using a complementary filter with a PI controller.

The VV was obtained by integrating the vertical acceleration. The vertical acceleration was calculated by the Euler angle transformation of the three-axis acceleration data [37]. Only the vertical acceleration component, $a_{z, g}$, was used, with $\mathrm{g}$ added to make the vertical acceleration equal to $0 \mathrm{~g}$ in the static state. The equations are as follows:

$$
\begin{gathered}
{\left[\begin{array}{l}
a_{x, g} \\
a_{y, g} \\
a_{z, g}
\end{array}\right]=\left[\begin{array}{ccc}
\cos \theta & \sin \theta \sin \varnothing & \sin \theta \cos \varnothing \\
0 & \cos \varnothing & -\sin \varnothing \\
-\sin \theta & \cos \theta \sin \varnothing & \cos \theta \cos \varnothing
\end{array}\right]\left[\begin{array}{l}
a_{x, l} \\
a_{y, l} \\
a_{z, l}
\end{array}\right],} \\
a_{v}=a_{z, g}+1 g
\end{gathered}
$$

In Equation (1), $a_{x, g}, a_{y, g}$, and $a_{z, g}$ represent the accelerations along the $\mathrm{x}^{-}, \mathrm{y}^{-}$, and $\mathrm{z}$-axes in the global coordinates, respectively; $a_{x, l}, a_{y, l}$, and $a_{z, l}$ represent the accelerations along the $x_{-}^{-}, y^{-}$, and z-axes 
in the local coordinates, respectively. $\varnothing$ (roll) and $\theta$ (pitch) represent the VAs in the frontal and sagittal planes, respectively. $a_{v}$ represents the vertical acceleration.

The VV can be obtained by integrating the vertical acceleration, but an integral accumulation error occurs when simple integration is performed. Lee et al. [38] used a conditional damping-based integration technique to remove this error. In their study, the vertical acceleration and jerk reference value were selected through trial and error; if a value greater than the reference value was entered, then integration was executed. In this study, we used a modified version of the above technique. Integration was executed using Simpson's rule when the vertical acceleration was $0.24 \mathrm{~g}$ or more. If the vertical acceleration was less than $0.24 \mathrm{~g}$, then the VV was initialized to $0 \mathrm{~m} / \mathrm{s}$ with an attenuation ratio of 0.9 . We selected a vertical acceleration of $0.24 \mathrm{~g}$ experimentally through trial and error. The estimated VV was lower than the actual VV because integration was not performed from the beginning owing to the conditional integration technique. The VV values calculated from the IMU sensor data during free-fall tests at heights of $1,1.5$, and $2 \mathrm{~m}$ were compared with those obtained from the 3D motion capture system, and a correction factor of 1.2 was used. The final VV calculation was performed as follows:

$$
v_{v, t}=\left\{\begin{array}{l}
\text { if } a_{v}>0.24 g, \\
v_{v, t-2}+1.2 g\left(a_{v, t-2}+4 a_{v, t-1}+a_{v, t}\right) \Delta t / 3 \\
\text { otherwise, } \\
v_{v, t-1} * 0.9
\end{array},\right.
$$

where $v_{v}$ represents the VV.

The VV calculated from the inertial data and that obtained from the 3D motion capture system were compared for the experiments involving humans to validate the VV calculated using the above equation. To confirm whether there were significant differences between the two sets of values, we performed a one-sample $t$-test $(p<0.05)$ using IBM SPSS Statistics 25 (IBM, New York, NY, USA). The results indicated that there were no significant differences between the two sets of values.

\subsection{Algorithm Development}

To set thresholds for the VA and VV to be used in the FFH detection algorithm, we used data collected from 10 randomly selected participants in Experiment 1. In a general receiver operating characteristic (ROC) curve, the $x$ - and $y$-axes represent specificity and sensitivity, respectively. In this study, a modified ROC curve was used in which the $x$ - and y-axes represented VV threshold and accuracy, respectively. Sensitivity, specificity, and accuracy were calculated as follows:

$$
\begin{gathered}
\text { Sensitivity }(\%)=\frac{T P}{T P+F N} \times 100, \\
\text { Specificity }(\%)=\frac{T N}{T N+F P} \times 100, \\
\text { Accuracy }(\%)=\frac{T P+T N}{T P+T N+F P+F N} \times 100,
\end{gathered}
$$

where $T P$ (true positive) is the number of FFHs detected as FFHs, $F N$ (false negative) is the number of FFHs detected as non-FFHs, TN(true negative) is the number of non-FFHs detected as non-FFHs, and $F P$ (false positive) is the number of non-FFHs detected as FFHs.

The threshold VA values were set to $25^{\circ}, 30^{\circ}, 35^{\circ}$, and $40^{\circ}$, and the threshold VV values were set from 1 to $3 \mathrm{~m} / \mathrm{s}$, with intervals of $0.1 \mathrm{~m} / \mathrm{s}$ for each VA threshold. Then, accuracy was calculated according to the threshold combinations of the VA and VV (Figure 6). Four threshold combinations of the VA and $\operatorname{VV}\left(25^{\circ}, 2.5 \mathrm{~m} / \mathrm{s} ; 30^{\circ}, 2.2 \mathrm{~m} / \mathrm{s} ; 35^{\circ}, 1.9 \mathrm{~m} / \mathrm{s}\right.$; and $\left.40^{\circ}, 1.8 \mathrm{~m} / \mathrm{s}\right)$ were obtained with $100 \%$ accuracy.

Data collected from the remaining 10 participants were used to select the best threshold combination among the four combinations. Table 3 presents the sensitivity, specificity, and accuracy levels obtained using the threshold combinations. 


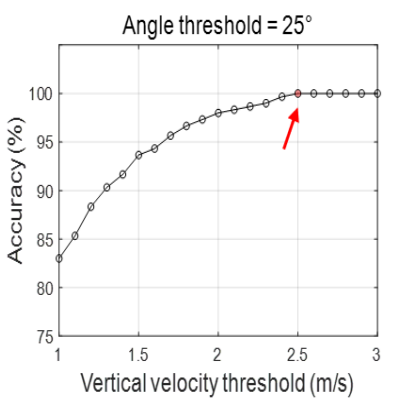

(a)

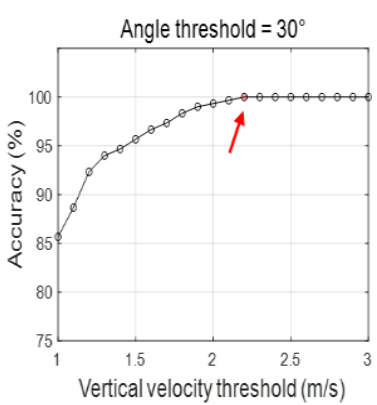

(b)

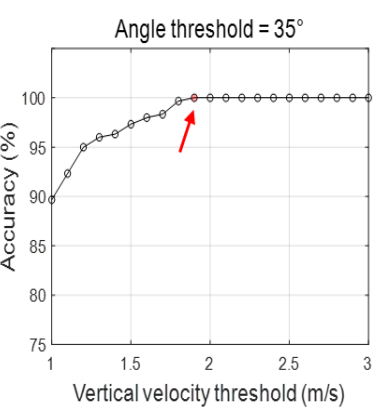

(c)

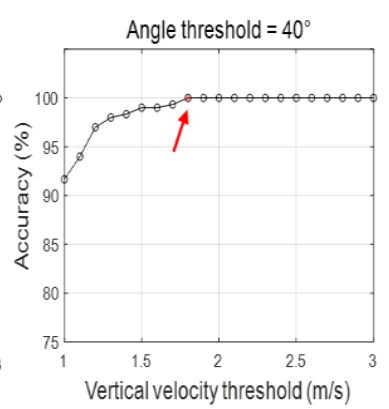

(d)

Figure 6. Accuracy determined using VA and VV thresholds.

Table 3. Sensitivity, specificity, and accuracy obtained using threshold combinations.

\begin{tabular}{ccccc}
\hline VA $\left({ }^{\circ}\right)$ & VV (m/s) & Sensitivity (\%) & Specificity (\%) & Accuracy (\%) \\
\hline 25 & 2.5 & 100 & 97.04 & 97.33 \\
30 & 2.2 & 100 & 99.63 & 99.67 \\
35 & 1.9 & 100 & 100 & 100 \\
40 & 1.8 & 100 & 100 & 100 \\
\hline
\end{tabular}

The lead time was determined to select one of the threshold combinations $\left(35^{\circ}, 1.9 \mathrm{~m} / \mathrm{s} ; 40^{\circ}\right.$, $1.8 \mathrm{~m} / \mathrm{s}$ ). The lead time is the interval between the collision time and the FFH detection time. It was calculated using the following equation:

$$
\text { Lead time }=\text { Collision time }- \text { FFH detection time. }
$$

Table 4 presents the lead times of FFHs according to the threshold combinations $\left(35^{\circ}, 1.9 \mathrm{~m} / \mathrm{s} ; 40^{\circ}\right.$, $1.8 \mathrm{~m} / \mathrm{s})$. The threshold combination with a longer lead time $\left(40^{\circ}, 1.8 \mathrm{~m} / \mathrm{s}\right)$ was selected as the final threshold combination for the FFH detection algorithm.

Table 4. Lead times according to threshold combinations.

\begin{tabular}{ccc}
\hline VA Threshold $\left(^{\circ}\right)$ & VV Threshold $(\mathrm{m} / \mathbf{s})$ & Lead Time $(\mathrm{ms})$ \\
\hline 35 & 1.9 & $326.7 \pm 41.7$ \\
40 & 1.8 & $333.7 \pm 45.8$ \\
\hline
\end{tabular}

An algorithm using only the VV was necessary as the algorithm containing the VA could not detect FFHs in which the VA did not change. In the algorithm using only the VV, the threshold was set to $5 \mathrm{~m} / \mathrm{s}$; this was greater than the maximum VV of non-FFHs, namely, $4.14 \mathrm{~m} / \mathrm{s}$. Figure 7 shows the flowchart of the final algorithm developed for detecting pre-impact FFHs.

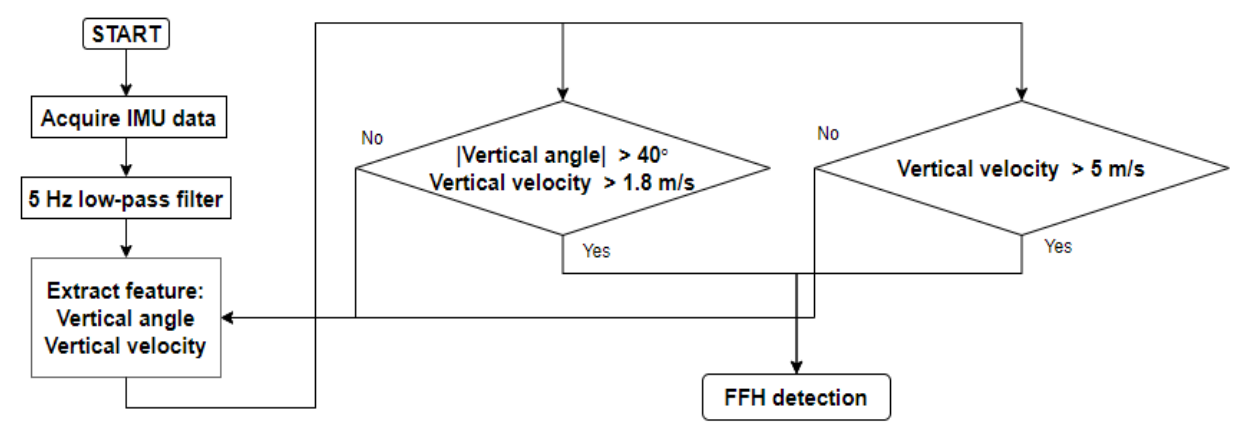

Figure 7. Flowchart of the threshold-based algorithm for detecting FFHs. 


\section{Results}

The algorithm was evaluated with the data from three different studies: human (Experiment 2), mannequin, and dynamics simulations. We achieved $100 \%$ sensitivity, $100 \%$ specificity, $100 \%$ accuracy, and a lead time of $301.8 \pm 87.8 \mathrm{~ms}$ for the human study of Experiment 2 .

In order to validate the results from both mannequin experiments and dynamics simulations, the VA and VV in $0.7 \mathrm{~m}$ jump and $0.7 \mathrm{~m}$ forward FFH were compared with those from human experiments (Table 5). An independent-sample $t$-test $(p<0.05)$ was conducted using IBM SPSS Statistics 25 to compare the data. In $0.7 \mathrm{~m}$ jump, no significant difference in the VA (roll) and VV was observed. However, a difference was found in the VA (pitch) because the upper body tended to bend over to maintain balance in human experiments. Furthermore, in $0.7 \mathrm{~m}$ forward FFH, a significant difference in the VA (roll), but not in the VA (pitch) and VV, was found. A slight rotation of the mannequin during forward FFH motion was found probably due to the uneven push.

Table 5. VA $\left(^{\circ}\right)$ and VV $(\mathrm{m} / \mathrm{s})$ determined from experiments involving humans and the mannequin and from dynamics simulations.

\begin{tabular}{cccc}
\hline & & $\mathbf{0 . 7} \mathbf{~}$ Jump & $\mathbf{0 . 7} \mathbf{~ m}$ Forward FFHs \\
\hline \multirow{3}{*}{ VA (roll) } & Human & $7.1 \pm 2.7$ & $11.6 \pm 5.4$ \\
& Dynamics simulation & $6.0 \pm 1.0$ & $25.8 \pm 7.6$ \\
& Human & $33.5 \pm 13.3$ & $87.8 \pm 8.5$ \\
VA (pitch) & Mannequin & $4.6 \pm 2.9$ & $81.5 \pm 3.1$ \\
& Dynamics simulation & 5.9 & 94.6 \\
\hline \multirow{2}{*}{ VV } & Human & $3.4 \pm 0.6$ & $4.7 \pm 0.4$ \\
& Mannequin & $3.3 \pm 0.1$ & $4.5 \pm 0.2$ \\
& Dynamics simulation & 3.5 & 4.7 \\
\hline
\end{tabular}

Statistical analysis could not be performed for the dynamics simulations. Some differences were observed for the VA in $0.7 \mathrm{~m}$ jump. Free fall from dynamics simulations differs from the actual human jump since humans tend to bend their upper body when jumping.

When evaluating the developed algorithm using the data from the mannequin experiments, we achieved $100 \%$ accuracy and a lead time of over $130 \mathrm{~ms}$. Table 6 presents the lead times obtained according to the motions performed in the mannequin experiments.

Table 6. Lead times (ms) during various motions in the mannequin experiments.

\begin{tabular}{ccc}
\hline Height $(\mathbf{m})$ & Vertical FFHs & Forward FFHs \\
\hline 2 & $176.0 \pm 37.7$ & $306.0 \pm 34.4$ \\
2.5 & $270.0 \pm 64.8$ & $378.0 \pm 44.0$ \\
3 & $368.0 \pm 20.4$ & $472.0 \pm 31.9$ \\
\hline
\end{tabular}

When evaluating the algorithm using the data from the dynamics simulations, we achieved $100 \%$ accuracy and a lead time of over $240 \mathrm{~ms}$. Table 7 presents the lead times obtained according to different motions in the dynamics simulations.

Table 7. Lead times (ms) according to different motions in the dynamics simulations.

\begin{tabular}{cccc}
\hline & $\mathbf{3 ~ \mathbf { ~ }}$ & $\mathbf{4 . 5} \mathbf{~ m}$ & $\mathbf{6 ~} \mathbf{~}$ \\
\hline Scaffold FFHs & 280 & 440 & 560 \\
Roof FFHs & 240 & 410 & 560 \\
Ladder FFHs & 460 & 640 & 780 \\
\hline
\end{tabular}




\section{Discussion}

Dogan et al. [23] developed an algorithm for detecting FFHs with 100\% accuracy using an IMU sensor. However, the study had no actual human motion data to evaluate the algorithm objectively, and it is difficult to apply the algorithm to wearable airbags because it was a post-impact FFH detection algorithm. Yang et al. [24] and Dzeng et al. [25] developed FFH detection algorithms based on the inertial data obtained from experiments with human participants, and they achieved relatively high FFH detection accuracies. However, these algorithms cannot be applied to wearable airbags because the number of participants and motions was too small, and the algorithms were not pre-impact FFH detection algorithms.

In this study, a sufficient amount of data was obtained from 40 healthy young males in the experiments, including 16 different types of motions. In addition, data on FFHs from great heights were obtained through mannequin experiments and dynamics simulations. An algorithm for detecting pre-impact FFHs was developed with 100\% accuracy based on the data obtained. The algorithm achieved sufficient lead time for wearable airbags to be inflated before collision with the floor. Table 8 shows a comparison of results from this study and those from existing studies.

Table 8. Comparison with other studies.

\begin{tabular}{ccccc}
\hline & Dogan et al. [23] (2019) & Yang et al. [24] (2014) & Dzeng et al. [25] (2017) & This Study \\
\hline \multirow{2}{*}{ Participants } & $\mathrm{X}$ & 2 males & 3 males, & 40 males \\
Sensor & IMU & IMU (waist) & IMU (back waist) & IMU (T7) \\
Method & Threshold & Machine learning & Threshold & Threshold \\
& (Acc RMS) & (SVM) & (Acc RMS) & (VA, VV) \\
Motions & 1 type & 6 types & 6 types & 16 types \\
Accuracy & (free fall) & (near-miss fall) & $(1 \mathrm{FFH})$ & $(1 \mathrm{FFH})$ \\
Lead time & $100 \%$ & $93.8 \%$ & $96.6 \%$ & $100 \%$ \\
\hline
\end{tabular}

\subsection{Lead Time}

When evaluating the developed algorithm using the data from Experiment 2, a lead time of $301.8 \pm 87.8 \mathrm{~ms}$ was obtained. This lead time was smaller than that obtained using the data from Experiment 1, and the standard deviation was larger. We observed that the VA changed slightly when the knee touched the ground first in FFHs, yielding a short lead time. These results were obtained because more participants touched their knees to the ground first during FFHs in Experiment 2 than in Experiment 1.

In the mannequin experiments, the lead time of forward FFHs was longer than that of vertical FFHs at the same height. In vertical FFHs, the VA did not change. Therefore, the VV had to exceed $5 \mathrm{~m} / \mathrm{s}$ to be detected as FFHs. Further, forward FFHs were detected when the VV exceeded $1.8 \mathrm{~m} / \mathrm{s}$ because the VA exceeded $40^{\circ}$. Detecting FFHs at low VVs caused FFHs to be detected faster, resulting in longer lead times.

In the dynamics simulations, the lead time obtained for the ladder FFHs was longer than those for the scaffold and roof FFHs. Ladder FFHs were detected as FFHs at a VV of $1.8 \mathrm{~m} / \mathrm{s}$ as the VA exceeded $40^{\circ}$, similar to forward FFHs in the mannequin experiments. However, other FFHs were detected as FFHs at a VV of $5 \mathrm{~m} / \mathrm{s}$ because the VA did not exceed $40^{\circ}$. Thus, the ladder FFHs had longer lead times.

The lead times obtained in the experiments involving humans, in the mannequin experiments, and in the dynamics simulations were $301.8 \pm 87.8 \mathrm{~ms}$, greater than $130 \mathrm{~ms}$, and greater than $240 \mathrm{ms,}$ respectively. The inflation time of the developed wearable airbag with a non-gunpowder inflator was approximately $100 \mathrm{~ms}$ [15]. If the present algorithm is applied to wearable airbags, the lead time is sufficient to inflate the airbags before collision with the floor. 


\subsection{Limitations}

Since FFHs from great heights could not be performed for safety issues in human experiments, mannequin experiments and dynamics simulations were performed. However, it was difficult to implement motion patterns that a human reacts in the actual FFH in mannequin experiments and dynamics simulations. Therefore, there was a difference between the actual FFH data and the experimental data. In addition, due to limited space and lack of equipment, non-FFH and FFH motions that could be performed were limited.

\section{Conclusions}

In this study, a threshold-based algorithm was developed for detecting pre-impact FFHs using only one IMU sensor. Forty healthy young males participated in two types of experiments. Data from 20 of the participants were used to develop the FFH detection algorithm, whereas data from the remaining 20 participants were used to evaluate the algorithm. In addition, mannequin experiments and dynamics simulations were performed to obtain inertial data on FFHs from great heights. The VA and VV were calculated using the inertial data from the sensor attached to the T7 and were used as parameters of the algorithm.

The algorithm achieved $100 \%$ accuracy in all the experiments. The lead times obtained during the experiments involving humans, the mannequin experiments, and the dynamics simulations were $301.8 \pm 87.8 \mathrm{~ms}$, greater than $130 \mathrm{~ms}$, and greater than $240 \mathrm{~ms}$, respectively. In the future, the algorithm will be further evaluated, improved using inertial data obtained from on-site testing, and applied to wearable airbags to significantly mitigate the damage caused by FFHs.

Author Contributions: H.J., B.K., and J.K. conceived, designed, and performed the experiments; H.J. and Y.K. wrote the paper and analyzed the data; and T.K. and Y.N. developed the IMU. All authors have read and agreed to the published version of the manuscript.

Funding: This research was supported by the Basic Science Research Program through the National Research Foundation of Korea (NRF), funded by the Ministry of Science and ICT (No. 2018R1D1A1B07048575), and the Technology Innovation Program (20006386), funded by the Ministry of Trade, Industry, and Energy (MOTIE, Korea).

Conflicts of Interest: The authors declare no conflict of interest. The funders had no role in the design of the study; in the collection, analyses, or interpretation of data; in the writing of the manuscript; or in the decision to publish the results.

\section{References}

1. Ministry of Employment and Labor of Korea. Industrial Accident Current Situation Analysis (Focus on Occupational Accident by Industrial Accident Compensation Act); Ministry of Employment and Labor of Korea: Sejong-si, Korea, 2018; 11-1490000-000022-10.

2. Socias-Morales, C.M.; Chaumont Menéndez, C.K.; Marsh, S.M. Fatal work-related falls in the United States, 2003-2014. Am. J. Ind. Med. 2018, 61, 204-215. [CrossRef] [PubMed]

3. Bureau of Labor Statistics, U.S. Department of Labor, the Economics Daily, Fatal Work-Related Falls to a Lower Level Increased 26 Percent from 2011 to 2016. Available online: https:/www.bls.gov/opub/ted/ 2018/fatal-work-related-falls-to-a-lower-level-increased-26-percent-from-2011-to-2016.htm (accessed on 3 March 2020).

4. Health and Safety Executive. Workplace Fatal Injuries in Great Britain, 2019. HSE Annual Statistics 3; Health and Safety Executive: London, UK, 2019.

5. United Nations' International Labor Organization. Top Ccauses of Global Construction Fatalities, and How to Avoid Site Risks. Available online: https://www.constructionweekonline.com/people/training/255830-top10-causes-of-construction-deaths-and-how-to-prevent-site-accidents (accessed on 4 March 2020).

6. Kaskutas, V.; Dale, A.M.; Lipscomb, H.; Evanoff, B. Fall prevention and safety communication training for foremen: Report of a pilot project designed to improve residential construction safety. J. Saf. Res. 2013, 44, 111-118. [CrossRef] [PubMed]

7. Marin, L.S.; Roelofs, C. Promoting construction supervisors' safety-efficacy to improve safety climate: Training intervention trial. J. Constr. Eng. M ASCE 2017, 143, 04017037. [CrossRef] 
8. Awlousi, I.; Marks, E.; Hallowell, M. Wearable technology for personalized construction safety monitoring and trending: Review of applicable devices. Automat. Constr. 2018, 85, 96-106. [CrossRef]

9. Lipscomb, H.J.; Schoenfisch, A.L.; Cameron, W.; Kucera, K.L.; Adams, D.; Silverstein, B.A. How well are we controlling falls from height in construction? Experiences of union carpenters in Washington State, 1989-2008. Am. J. Ind. Med. 2014, 57, 69-77. [CrossRef]

10. Yang, K.; Ahn, C.R.; Vuran, M.C.; Aria, S.S. Semi-supervised near-miss fall detection for ironworkers with a wearable inertial measurement unit. Automat. Constr. 2016, 68, 194-202. [CrossRef]

11. Chi, C.-F.; Chang, T.-C.; Ting, H.-I. Accident patterns and prevention measures for fatal occupational falls in the construction industry. Appl. Ergon. 2005, 36, 391-400. [CrossRef]

12. Bobick, T.G.; McKenzie, E.A., Jr.; Kau, T.-Y. Evaluation of guardrail systems for preventing falls through roof and floor holes. J. Saf. Res. 2010, 41, 203-211. [CrossRef]

13. Lee, C.; Porter, K.M. Suspension trauma. Emerg. Med. J. 2007, 24, 237-238. [CrossRef]

14. Dong, X.S.; Largay, J.A.; Choi, S.D.; Wang, X.; Cain, C.T.; Romano, N. Fatal falls and PFAS use in the construction industry: Findings from the NIOSH FACE reports. Accid. Anal. Prev. 2017, 102, 136-143. [CrossRef]

15. Tamura, T.; Yoshimura, T.; Sekine, M.; Uchida, M.; Tanaka, O. A Wearable Airbag to Prevent Fall Injuries. IEEE Trans. Inf. Technol. Biomed. 2009, 13, 910-914. [CrossRef] [PubMed]

16. Zhong, Z.; Chen, F.; Zhai, Q.; Fu, Z.; Ferreira, J.P.; Liu, Y.; Yi, J.; Liu, T. A real-time pre-impact fall detection and protection system. In Proceedings of the 2018 IEEE/ASME International Conference on Advanced Intelligent Mechatronics (AIM), Auckland, New Zealand, 9-12 July 2018; pp. 1039-1044.

17. Thanh, P.V.; Tran, D.T.; Nguyen, D.C.; Anh, N.D.; Dinh, D.N.; El-Rabaie, S.; Sandrasegara, K. Development of a real-time, simple and high-accuracy fall detection system for elderly using 3-DOF accelerometers. Arab. J. Sci. Eng. 2019, 44, 3329-3342. [CrossRef]

18. Ahn, S.; Choi, D.; Kim, J.; Kim, S.; Jeong, Y.; Jo, M.; Kim, Y. Optimization of a pre-impact fall detection algorithm and development of hip protection airbag system. Sens. Mater. 2018, 30, 1743-1752. [CrossRef]

19. Jeong, Y.; Ahn, S.; Kim, J.; Kim, S.; Koo, B.; Ryu, J.; Kim, Y. Impact attenuation of the soft pads and the wearable airbag for the hip protection in the elderly. Int. J. Precis. Eng. Manuf. 2019, 20, 273-283. [CrossRef]

20. Han, S.; Lee, S.; Peña-Mora, F. Vision-based detection of unsafe actions of a construction worker: Case study of ladder climbing. J. Comput. Civ. Eng. 2013, 27, 635-644. [CrossRef]

21. Shrestha, K.; Shrestha, P.P.; Bajracharya, D.; Yfantis, E.A. Hard-hat detection for construction safety visualization. J. Constr. Eng. (Hindawi) 2015, 721380. [CrossRef]

22. Fang, W.; Zhong, B.; Zhao, N.; Love, P.E.D.; Luo, H.; Xue, J.; Xu, S. A deep learning-based approach for mitigating falls from height with computer vision: Convolutional neural network. Adv. Eng. Inform. 2019, 39, 170-177. [CrossRef]

23. Dogan, O.; Akcamete, A. Detecting falls-from-height with wearable sensors and reducing consequences of occupational fall accidents leveraging IoT. In Advances in Informatics and Computing in Civil and Construction Engineering; Springer: Cham, Switzerland, 2019; pp. 207-214.

24. Yang, K.; Aria, S.; Ahn, C.; Stentz, T.L. Automated detection of near-miss fall incidents in iron workers using inertial measurement units. Construction Research Congress. In Proceedings of the Construction Research Congress 2014, Atlanta, GA, USA, 19-21 May 2014.

25. Dzeng, R.-J.; Fang, Y.-C.; Chen, I.-C. A feasibility study of using smartphone built-in accelerometers to detect fall portents. Automat. Constr. 2014, 38, 74-86. [CrossRef]

26. Casilari, E.; Santoyo- Ramón, J.A.; Cano-García, J.M. UMAFall: A Multisensor Dataset for the Research on Automatic Fall Detection. Procedia Comput. Sci. 2017, 110, 32-39. [CrossRef]

27. Sucerquia, A.; López, J.D.; Vargas-Bonilla, J.F. SisFall: A fall and movement dataset. Sensors 2017, $19,198$. [CrossRef]

28. Martínez-Villaseñor, L.; Ponce, H.; Brieva, J.; Moya-Albor, E.; Núñez-Martínez, J.; Peñafort-Asturiano, C. UP-Fall Detection Dataset: A Multimodal Approach. Sensors 2019, 19, 1988. [CrossRef]

29. Tong, L.; Song, Q.; Ge, Y.; Liu, M. HMM-Based Human Fall Detection and Prediction Method Using Tri-Axial Accelerometer. IEEE Sens. J. 2013, 13, 1849-1856. [CrossRef]

30. Zhen, T.; Mao, L.; Wang, J.; Gao, Q. Wearable Preimpact Fall Detector using SVM. In Proceedings of the Tenth International Conference on Sensing Technology, Nanjing, China, 11-13 November 2016; pp. 1-6. [CrossRef] 
31. Wu, G.; Xue, S. Portable Preimpact Fall Detector with Inertial Sensors. IEEE Trans. Neural Syst. Rehabil. Eng. 2008, 16, 178-183. [PubMed]

32. Nyan, M.N.; Tay, F.E.H.; Murugasu, E. A wearable system for pre-impact fall detection. J. Biomech. 2008, 41, 3475-3481. [CrossRef] [PubMed]

33. Sabatini, A.M.; Ligorio, G.; Mannini, A.; Genovese, V.; Pinna, L. Prior-to- and Post-Impact Fall Detection Using Inertial and Barometric Altimeter Measurements. IEEE Trans. Neural Syst. Rehabil. Eng. 2016, 24, 774-783. [CrossRef]

34. Jung, H.; Koo, B.; Kim, J.; Kim, T.; Nam, Y.; Kim, Y. Enhanced algorithm for the detection of preimpact fall for wearable airbags. Sensors 2020, 20, 1277. [CrossRef]

35. InvenSense, MPU-9250 Nine-Axis MEMS Motion Tracking Device. Available online: https://www.invensense. com/products/motion-tracking/9-axis/mpu-9250/ (accessed on 24 April 2020).

36. Zlatar, T.; Lago, E.M.G.; Soares, W.A.; Baptista, J.S.; Junior, B.B. Falls from height: Analysis of 114 cases. Production 2019, 29, e20180091. [CrossRef]

37. Lee, S.C.; Hong, S.K.; Jung, S.R. Estimation for the vertical speed of helicopter using MEMS inertial sensor. In Proceedings of the Autumn Conference of The Korean Society for Aeronautical \& Space Sciences, Wonju, Korea, 16-18 April 2014; pp. 1422-1425.

38. Lee, J.K.; Robinovitch, S.N.; Park, E.J. Inertial sensing-based pre-impact detection of falls involving near-fall scenarios. IEEE Trans. Neural Syst. Rehabil. Eng. 2015, 23, 258-266. [CrossRef]

(C) 2020 by the authors. Licensee MDPI, Basel, Switzerland. This article is an open access article distributed under the terms and conditions of the Creative Commons Attribution (CC BY) license (http://creativecommons.org/licenses/by/4.0/). 